\title{
Short tandem repeat number estimation from paired-end reads for multiple individuals by considering coalescent tree
}

\author{
Kaname Kojima ${ }^{1}$, Yosuke Kawai ${ }^{1}$, Naoki Nariai ${ }^{2}$, Takahiro Mimori ${ }^{1}$, Takanori Hasegawa ${ }^{1}$
} and Masao Nagasaki ${ }^{*}$

From 11th International Symposium on Bioinformatics Research and Applications (ISBRA '15)

Norfolk, VA, USA. 7-10 June 2015

\begin{abstract}
Background: Two types of approaches are mainly considered for the repeat number estimation in short tandem repeat (STR) regions from high-throughput sequencing data: approaches directly counting repeat patterns included in sequence reads spanning the region and approaches based on detecting the difference between the insert size inferred from aligned paired-end reads and the actual insert size. Although the accuracy of repeat numbers estimated with the former approaches is high, the size of target STR regions is limited to the length of sequence reads. On the other hand, the latter approaches can handle STR regions longer than the length of sequence reads. However, repeat numbers estimated with the latter approaches is less accurate than those with the former approaches.

Results: We proposed a new statistical model named coalescentSTR that estimates repeat numbers from paired-end read distances for multiple individuals simultaneously by connecting the read generative model for each individual with their genealogy. In the model, the genealogy is represented by handling coalescent trees as hidden variables, and the summation of the hidden variables is taken on coalescent trees sampled based on phased genotypes located around a target STR region with Markov chain Monte Carlo. In the sampled coalescent trees, repeat number information from insert size data is propagated, and more accurate estimation of repeat numbers is expected for STR regions longer than the length of sequence reads.

For finding the repeat numbers maximizing the likelihood of the model on the estimation of repeat numbers, we proposed a state-of-the-art belief propagation algorithm on sampled coalescent trees.

Conclusions: We verified the effectiveness of the proposed approach from the comparison with existing methods by using simulation datasets and real whole genome and whole exome data for HapMap individuals analyzed in the 1000 Genomes Project.
\end{abstract}

Keywords: High-throughput sequencing, Short tandem repeat, Coalescent theory

\footnotetext{
*Correspondence: nagasaki@megabank.tohoku.ac.jp

${ }^{1}$ Tohoku Medical Megabank Organization, Tohoku University, 2-1,

Seiryo-machi, Aoba-ku, 980-8573, Sendai, Japan

Full list of author information is available at the end of the article
} 


\section{Background}

The progress of high-throughput sequencing (HTS) technologies enables the variant detection of each individual in genome-wide scale in practical time and with reasonable cost. From HTS data, various types of single nucleotide variant (SNV) calling methods have been proposed [1-4], and SNVs for more than a thousand of individuals were accurately detected [5]. However, unlike SNVs, we still have difficulty in accurately detecting structural variations such as genome insertions, genome deletions, short tandem repeat (STR) number polymorphisms, and copy number variations, especially from low coverage HTS data [6].

Some repeat number polymorphisms are associated with various disease phenotypes such as CAG repeats in the Huntingtin gene with Huntington's disease [7]. From HTS data, several approaches such as lobSTR and RepeatSeq $[8,9]$ have been proposed for the estimation of repeat numbers in STR regions by directly counting repeat patterns in sequence reads spanning the regions. In these approaches, the accuracy on both the detection of STR variants and estimated repeat numbers is high. Another strategy is to use paired-end reads aligned to the flanking regions of the target STR region in the reference genome [10]. Insert size inferred from the aligned paired-end reads is longer than its actual size if the repeat number is smaller than that in the reference genome. On the other hand, the inferred insert size is shorter if the repeat number is larger. By detecting the difference between the inferred and actual insert size, repeat numbers are estimated. Since insert size is generally longer than sequence reads, this strategy can be used for estimating repeat numbers for relatively long STR regions that cannot be handled by the strategy counting repeat patterns in sequence reads. However, repeat numbers estimated from insert size data are less accurate than those from the strategy counting repeat patterns directly in the sequence reads, especially for low coverage HTS data.

We proposed a new statistical model named coalescentSTR that estimates repeat numbers for multiple individuals simultaneously from paired-end read distances by connecting the read generative model for each individual with their genealogy. In the model, the genealogy is represented with coalescent trees, which describe the ancestral history of multiple individuals on a local genome region backwards in time [11-13]. By considering the change in repeat numbers in coalescent trees in a natural manner, more accurate estimation of repeat numbers is expected. For the estimation of repeat numbers in the model, coalescent trees handled as hidden variables are sampled with Markov chain Monte Carlo (MCMC) according to phased genotypes around a target STR region. We proposed a new belief propagation method that calculates the loopy belief propagation [14] and the mixed-product belief propagation [15] by taking the summation on the sampled coalescent trees. By using the proposed belief propagation, approximated maximum configuration of repeat numbers in the model are searched for the estimation of repeat numbers.

In a simulation study, we used synthetically generated HTS data for STR regions mostly longer than read length, and showed the effectiveness of our model from the comparison with other existing methods, especially in handling more individuals. The effectiveness of our approach is also verified from the analysis of real whole exome data of HapMap JPT individuals and whole genome sequencing (WGS) data of HapMap CEU and GBR individuals analyzed in the 1000 Genomes Project (1KGP).

\section{Method}

We describe a model considering insert size of pairedend reads for one individual and its extension to consider multiple individuals based on their unobserved genealogy. Procedures for the repeat number estimation are then explained.

\section{Repeat number estimation from paired-end read distance}

We consider a statistical model that estimates repeat number in an STR region from paired-end read distance for one individual. We hereafter call the model a basic model. Let $s^{(d)}$ be the start position of the forward read of the $d$ th aligned read pair. We also let $e^{(d)}$ be the end position of the reverse read of the $d$ th aligned read pair. The insert size of the $d$ th read pair or the length of the DNA fragment from which the read pair was generated is given by $e^{(d)}-s^{(d)}$, and we denote the insert size $e^{(d)}-s^{(d)}$ as $l^{(d)}$.

If an $x$ bp insertion variant exists between $s^{(d)}$ and $e^{(d)}$ in the genome of an individual, $l^{(d)}$ is $x$ bp shorter than the actual insert size of the $d$ th read pair. On the other hand, if an $x$ bp deletion variant exists between $s^{(d)}$ and $e^{(d)}, l^{(d)}$ is $x$ bp longer than its actual insert size. By detecting the difference between $l^{(d)}$ and the actual insert size, the basic model estimates repeat numbers. Let $u, n_{r}, n_{1}$, and $n_{2}$ be the length of a repeat pattern, the repeat number in reference genome, the repeat number on haplotype 1 , and the repeat number on haplotype 2 , respectively. If the DNA fragment for the $d$ th read pair spans the STR region in haplotype 1 , its actual insert size is given by $l^{(d)}+u \cdot\left(n_{1}-n_{r}\right)$, and hence the probability of $l^{(d)}$ is given by $\mathcal{F}\left(l^{(d)}+u \cdot\left(n_{1}-n_{r}\right)\right)$, where $\mathcal{F}$ is the insert size distribution of the sequence data. If the start or end position of the DNA fragment is in the inside of the STR region, the DNA fragment cannot be used for estimating repeat numbers. Thus, $l^{(d)}$ must be longer than $e_{m}-s^{(d)}$, where $e_{m}$ is the end position of the STR region in the reference genome. We also exclude the DNA fragment longer than $K$, i.e., $\mathcal{F}(l)$ takes 0 for $l>K$, where $K$ is a sufficiently large number and is set to $2,000 \mathrm{bp}$ in our study. The gen- 
erative probability of $l^{(d)}$ is represented by normalizing $\mathcal{F}$ as follows:

$P\left(l^{(d)} \mid n\right)=\left\{\begin{array}{cl}\frac{\mathcal{F}\left(l^{(d)}+u \cdot\left(n-n_{r}\right)\right)}{N\left(s^{(d)}, n\right)} & \text { if } l^{(d)}>e_{m}-s^{(d)} \& l^{(d)} \leq K-u \cdot\left(n-n_{r}\right), \\ 0 & \text { otherwise }\end{array}\right.$

where $N(s, n)$ is the normalization factor given by

$$
N(s, n)=\sum_{l=e_{m}-s+1}^{K-u \cdot\left(n-n_{r}\right)} \mathcal{F}\left(l+u \cdot\left(n-n_{r}\right)\right)
$$

Since each read pair is generated from one of two DNA sequences in equal probability, the likelihood of $l^{(d)}$ is represented by

$$
\prod_{d=1}^{D} P\left(l^{(d)} \mid n_{1}, n_{2}\right)=\prod_{d=1}^{D} \frac{1}{2}\left(P\left(l^{(d)} \mid n_{1}\right)+P\left(l^{(d)} \mid n_{2}\right)\right),
$$

where $D$ is the number of read pairs. We consider the maximum and minimum repeat numbers $n_{\max }$ and $n_{\min }$ and search the pair of $n_{i 1}$ and $n_{i 2}$ in $\left\{n_{\min }, \ldots, n_{\max }\right\} \times$ $\left\{n_{\min }, \ldots, n_{\max }\right\}$ maximizing Eq. (1), which requires $O\left(\left(n_{\max }-n_{\min }+1\right)^{2}\right)$ time. The computational time in the basic model is mainly taken by the calculation of the normalization factor $N(s, n)$, which requires $O\left(D\left(n_{\max }-\right.\right.$ $\left.\left.n_{\min }+1\right) K\right)$ time in a naïve way. Thus, we propose an algorithm that calculates $N(s, n)$ more efficiently by considering the following two recurrence formulae of $N(s, n)$ :

$$
\begin{aligned}
& N(s+1, n)=N(s, n)+\mathcal{F}\left(e_{m}-s+u \cdot\left(n-n_{r}\right)\right) \\
& N(s, n+1)=N(s, n)-\sum_{l=e_{m}-s+1}^{e_{m}-s+u} \mathcal{F}\left(l+u \cdot\left(n-n_{r}\right)\right) .
\end{aligned}
$$

By using the above recurrence formulae, $N(s, n)$ is calculated for $s \in\left\{s_{m}-K, s_{m}-1\right\}$ and $n \in\left\{n_{\min }, \ldots, n_{\max }\right\}$, where $s_{m}$ is the start position of the STR region. Since the repeat pattern size $u$ is usually less than or equal to four and can be considered as a constant, the calculation of $N(s, n)$ requires $O\left(\left(n_{\max }-n_{\min }+1\right) K\right)$ time, which is smaller than that required in the naïve way.

\section{Repeat number estimation considering genealogy of multiple individuals}

DNA sequences are inherited from parents to offspring, and single base substitutions occur in the inheritance with mutation rate of around $2.0 \times 10^{-8}$ [16]. Repeat numbers in STR regions also change or mutate in the inheritance from a parent to its offspring with rate ranging usually from $1.0 \times 10^{-4}$ to $1.0 \times 10^{-3}$ [17]. From the phased genotypes around an STR region of interest for multiple individuals, we consider their genealogy around the region by using coalescent tree [11-13]. Coalescent tree is a binary tree in which leaves represent the current haplotypes and internal nodes represent past coalescent events of the haplotypes. For each coalescent event, two linages are involved, and cases involving more than two lineages are not considered in our model. The length of each edge in the tree represents time between coalescent events.

We propose a new statistical model named coalescentSTR that uses coalescent trees estimated from phased genotypes around an STR region to connect the basic models of multiple individuals for more accurate estimation of their repeat numbers. Repeat numbers in the STR region obey the estimated coalescent tree. Thus, given nearby phased genotypes $V$, we consider the prior distribution of repeat numbers via coalescent trees estimated from $V$ and model insert size inferred from paired-end reads in the following formula. Let $l_{i}^{(d)}$ be an insert size of the $d$ th read pair for the $i$ th individual. We also denote $n_{i_{1}}$ and $n_{i_{2}}$ as repeat numbers of haplotype 1 and haplotype 2 for the $i$ th individual, respectively. We represent the likelihood of insert size $l_{i}^{(d)}$ as

$P(L, N \mid V)=\prod_{i=1}^{I} \prod_{d=1}^{D_{i}} P\left(l_{i}^{(d)} \mid n_{i_{1}}, n_{i_{2}}\right) \sum_{g} P(N \mid g) P(g \mid V)$,

where $I$ is the number of individuals, $L$ is a set of $l_{i}^{(d)}, N$ is a set of $n_{i_{1}}$ and $n_{i_{2}}, D_{i}$ is the number of read pairs for individual $i$, and $g$ is a coalescent tree. The first term in the right hand of Eq. (2) is given by the likelihood function of the basic model. In the second term, repeat numbers are connected by coalescent tree $g$ as

$P(N \mid g)=\sum_{n_{c_{1}}=n_{\min }}^{n_{\max }} \ldots \sum_{n_{c_{I-1}}=n_{\min }}^{n_{\max }} \prod_{v \in C_{g}} \prod_{u \in o_{v \mid g}} P\left(n_{u} \mid n_{v}, t_{v, u \mid g} ; \mu_{s}\right)$,

where $C_{g}$ is a set of internal nodes $c_{1}, \ldots, c_{I-1}$ in $g, o_{v \mid g}$ is a set of offspring nodes of $v$ in $g, t_{v, u \mid g}$ is coalescent time from node $v$ to $u$ in $g$, and $n_{v}$ is a repeat number in node $v$. Note that the size of $o_{v \mid g}$ is two. $P\left(n_{u} \mid n_{v}, t_{v, u \mid g} ; \mu_{s}\right)$ represents the change of repeat numbers from parent node $v$ to its offspring node $u$ in time $t_{v, u \mid g}$ with mutation rate $\mu_{s}$. For the change of repeat numbers, we consider the stepwise model [18], where repeat numbers change at most one in one generation with mutation rate $\mu_{s}$. With the Brownian motion approximation to the stepwise model [19], $P\left(n_{u} \mid n_{v}, t_{v, u \mid g} ; \mu_{s}\right)$ is given by

$$
P\left(n_{u} \mid n_{v}, t_{v, u \mid g} ; \mu_{s}\right)=\min \left\{1, \mathcal{N}\left(n_{u} ; n_{v}, N_{e} \cdot \mu_{s} \cdot t_{v, u \mid g}\right)\right\}
$$

where $\mathcal{N}$ represents the normal distribution and $N_{e}$ is the effective population size. $P(g \mid V)$ represents the probability of coalescent tree $g$ given nearby phased genotypes 
$V$. Since it is infeasible to calculate the summation in all possible coalescent trees $g$ in Eq. (2), we sample a set of coalescent trees $\mathcal{G}$ from the phased genotypes $V$ with MCMC $[13,20]$, and calculate the summation only on $g \in \mathcal{G}:$

$$
P(L, N \mid V)=\prod_{i=1}^{I} \prod_{d=1}^{D_{i}} P\left(l_{i}^{(d)} \mid n_{i_{1}}, n_{i_{2}}\right) \sum_{g \in \mathcal{G}} P(N \mid g) .
$$

For sampling with MCMC, burn-in period, period between samples, and the number of samples are respectively set to 50,000,100, and 100 in our study.

\section{Estimation of repeat numbers in coalescentSTR}

In coalescentSTR, repeat numbers are estimated by finding $N$ maximizing Eq. (4):

$$
\hat{N}=\arg \max _{N} \prod_{i=1}^{I} \prod_{d=1}^{D_{i}} P\left(l_{i}^{(d)} \mid n_{i_{1}}, n_{i_{2}}\right) \sum_{g \in \mathcal{G}} P(N \mid g)
$$

The calculation of the exact value of $\hat{N}$ is known as the marginal MAP problem and NP-hard even when the model structure including other hidden variables is a tree [15]. If the number of trees in $\mathcal{G}$ is one, i.e., the summation in $g$ is not considered, the mixed-product belief propagation (mixed-product BP) [15] can be applied to obtain an approximated solution of $\hat{N}$. However, if the summation in $g$ is considered, the mixed-product belief propagation cannot be applied directly, and a new algorithm is required for the solution. Here, we propose a new belief propagation algorithm named multiple-tree belief propagation (multiple-tree BP), which considers belief propagation in multiple trees. Given messages to variables in $N \backslash\left\{n_{i 1}\right\}$, we consider message passing from variables in $N \backslash\left\{n_{i 1}\right\}$ to $n_{i 1}$. In multiple-tree BP, message to $n_{i 1}$ on each tree is calculated independently. Since message passing can be calculated exactly on tree structures by the belief propagation, a message to $n_{i 1}$ can be obtained by taking the summation of messages to $n_{i 1}$ from trees. Multiple-tree BP is extended to the loopy belief propagation (loopy BP) [14] and mixed-product BP. We first consider multiple-tree BP for loopy BP, and then describe its extension to mixed-product BP later. A message from internal node $v$ in coalescent tree $g$ to its parent $p_{v \mid g}$ is given by

$m_{v \rightarrow p_{v \mid g}}\left(n_{p_{v \mid g}}\right)=\sum_{n_{v}=n_{\min }}^{n_{\max }} P\left(n_{u} \mid n_{v}, t_{v, u \mid g} ; \mu_{s}\right) \cdot m_{o_{1} \rightarrow v}\left(n_{v}\right) \cdot m_{o_{2} \rightarrow v}\left(n_{v}\right)$,

where $o_{1}$ and $o_{2}$ are offspring of $v$. On the other hand, a message from a leaf node $i_{1}$ to its parent $p_{i_{1} \mid g}$ in coalescent tree $g$ is given by $m_{i_{1} \rightarrow p_{i_{1} \mid g}}\left(n_{p_{i_{1} \mid g}}\right)=\sum_{n_{i_{1}}=n_{\min }}^{n_{\max }} P\left(n_{i_{1}} \mid n_{p_{i_{1} \mid g}}, t_{p_{i_{1} \mid g}} ; \mu_{s}\right) \cdot m_{i_{2} \rightarrow i_{1}}\left(n_{i_{1}}\right)$

where $m_{i_{2} \rightarrow i_{1}}\left(n_{i_{1}}\right)$ is a message from $n_{i_{2}}$ to $n_{i_{1}}$. A message from internal node $v$ to one of its offspring $o_{1}$ is given by

$m_{v \rightarrow o_{1}}\left(n_{o_{1}}\right)=\sum_{n_{v}=n_{\min }}^{n_{\max }} P\left(n_{i_{1}} \mid n_{p_{i_{1} \mid g}}, t_{p_{i_{1} \mid g}, i_{1}} ; \mu_{s}\right) \cdot m_{p_{v \mid g} \rightarrow v}\left(n_{v}\right) \cdot m_{o_{2} \rightarrow v}\left(n_{v}\right)$,

where $o_{2}$ is a sibling of $o_{1}$. A message from $n_{i_{1}}$ to $n_{i_{2}}$ is calculated by

$m_{i_{1} \rightarrow i_{2}}\left(n_{i_{2}}\right)=\sum_{n_{i_{1}}=n_{\min }}^{n_{\max }} \prod_{d=1}^{D_{i}} P\left(l_{i}^{(d)} \mid n_{i_{1}}, n_{i_{2}}\right) \cdot \sum_{g \in \mathcal{G}} m_{p_{i_{1} \mid g} \rightarrow i_{1}}\left(n_{i_{1}}\right)$.

Figure 1 illustrates the flow of the above messages. For the extension to mixed-product BP, the message from $n_{i_{1}}$ to $n_{i_{2}}$ in Eq. (6) is replaced with

$m_{i_{1} \rightarrow i_{2}}\left(n_{i_{2}}\right)=\max _{n_{i_{1}}}\left\{\prod_{d=1}^{D_{i}} P\left(l_{i}^{(d)} \mid n_{i_{1}}, n_{i_{2}}\right) \sum_{g \in \mathcal{G}} m_{p_{i_{1} \mid g} \rightarrow i_{1}}\left(n_{i_{1}}\right)\right\}$,

and Eq. (5) is replaced with

$\tilde{m}_{i_{1} \rightarrow p_{\left\{i_{1} \mid g\right\}}}\left(n_{p\left\{i_{1} \mid g\right\}}\right)=\sum_{n_{i_{1}} \in \boldsymbol{n}_{i_{1}}} P\left(n_{i_{1}}, n_{p\left\{i_{1} \mid g\right\}} ; \mu_{s}, t_{p_{\left\{i_{1} \mid g\right\}}, i_{1}}\right) \cdot m_{i_{2} \rightarrow i_{1}}\left(n_{i_{1}}\right)$,

where $\boldsymbol{n}_{i_{1}}$ is a set of $n_{i_{1}}$ maximizing $m_{i_{2} \rightarrow i_{1}}\left(n_{i_{1}}\right)$. $\sum_{g \in \mathcal{G}} m_{p_{i_{1} \mid g} \rightarrow i_{1}}\left(n_{i_{1}}\right)$. After some iterations of mixedproduct BP, $\hat{n}_{i_{1}} \in \hat{N}$ is obtained by $\arg \max _{n_{i_{1}}} m_{i_{2} \rightarrow i_{1}}\left(n_{i_{1}}\right)$. $\sum_{g \in \mathcal{G}} m_{p_{i_{1} \mid g} \rightarrow i_{1}}\left(n_{i_{1}}\right)$. We first calculate loopy BP in ten cycles and then calculate mixed-product BP in ten cycles using messages from loopy BP as initial values. Empirically, the above procedure provides better $\hat{N}$ than only considering mixed-product BP.

\section{Selection of STR mutation rate}

Messages from loopy BP is used to calculate the following value:

$\sum_{i=1}^{I}\left(\sum_{n_{i_{1}}=n_{\min }}^{n_{\max }} \bar{m}_{i_{2} \rightarrow i_{1}}\left(n_{i_{1}}\right) \cdot \bar{m}_{\mathcal{G} \rightarrow i_{1}}\left(n_{i_{1}}\right)+\sum_{n_{i_{2}}=n_{\min }}^{n_{\max }} \bar{m}_{i_{1} \rightarrow i_{2}}\left(n_{i_{2}}\right) \cdot \bar{m}_{\mathcal{G} \rightarrow i_{2}}\left(n_{i_{2}}\right)\right)$,

where $\bar{m}_{i_{2} \rightarrow i_{1}}\left(n_{i_{1}}\right)$ is $m_{i_{2} \rightarrow i_{1}}\left(n_{i_{1}}\right)$ normalized to have a sum of one and $\bar{m}_{\mathcal{G} \rightarrow i_{1}}\left(n_{i_{1}}\right)$ is a message to $n_{i_{1}}$ from coalescent trees $\sum_{g \in \mathcal{G}} m_{p_{i_{1} \mid g}}\left(n_{i_{1}}\right)$ normalized to have a sum of one. We consider that messages from paired-end reads to $n_{i_{1}}$ or $n_{i_{2}}$ and from coalescent trees to $n_{i_{1}}$ or $n_{i_{2}}$ are similar to each other if the STR mutation rate is proper. The value in 


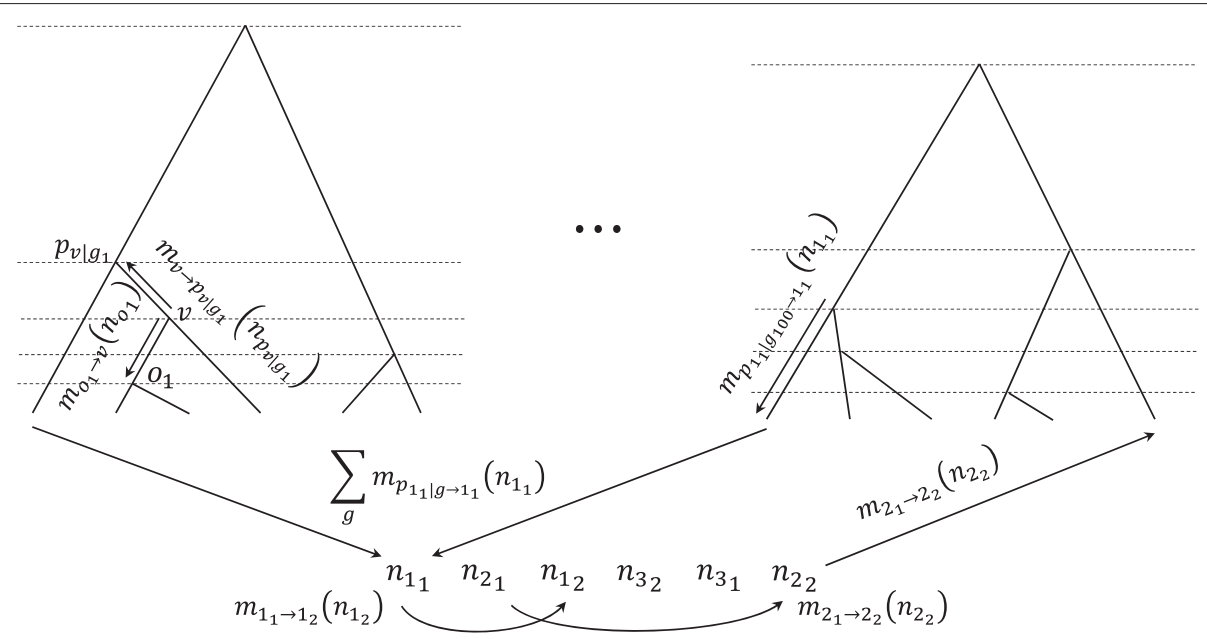

Fig. 1 Coalescent trees on three individuals and messages in the belief propagation. The flow of messages from $v$ to $p_{v \mid g_{1}}$, from $v$ to its offspring $o_{1}$, from leaf $1_{1}$ to leaf $1_{2}$, from leaf $2_{1}$ to leaf $2_{2}$, from $p_{1} \mid g_{100}$ to leaf $1_{1}$, and from trees to leaf $1_{1}$ are illustrated

Eq. (7) is designed to take higher value if those messages are more similar to each other. We consider several STR mutation rates and select the rate with the highest value given by Eq. (7).

\section{Results and discussion}

\section{Simulation analysis}

Given a target STR region, we first synthetically generated repeat numbers of the STR region and nearby phased genotypes for $2 I$ haplotypes as follows:

- Generate a coalescent tree for $2 I$ haplotypes with an algorithm in [18] under the assumption of a constant effective population size.

- Obtain phased genotypes at 1,000 bp upstream and downstream positions of the STR region based on the generated coalescent tree and a specified single base substitution rates.

- Obtain repeat numbers based on the generated coalescent tree by considering the stepwise model with a specified STR mutation rate.

The effective population size was set to 10,400 [21], and single base substitution rates on transition and transversion were set to $5.5 \times 10^{-8}$ and $1.2 \times 10^{-8}$, respectively as in [16]. For the STR region, we considered TTTC repeat region at chr7:127898719-127898787 in the human reference genome (GRCh37) from tandem repeat regions detected by Tandem Repeats Finder [22]. The reference repeat number, the repeat number in the reference genome for the region, is 17 . We obtained synthetically generated diploid genome sequences for each individual by editing GRCh37 chromosome 7 sequence according to repeat numbers in the region and phased genotypes around the region generated by the above procedures.
The following settings were considered for the number of individuals and mutation rate:

- Five types of the numbers of individuals: 5, 10, 20, 50, and 100.

- Two types of STR mutation rates: $1.00 \times 10^{-3}$ and $2.73 \times 10^{-4}$. The former rate is an estimated STR mutation rate for tetranucleotide repeats, and the latter for dinucleotide repeats in human [17].

From each edited diploid genome pair, paired-end read reads with length of $100 \mathrm{bp}$ and $0.1 \%$ base substitution errors were generated in FASTQ format. Insert size of each read pair is normally distributed with mean $350 \mathrm{bp}$ and standard deviation $50 \mathrm{bp}$. For the read coverage for each individual, $20 \times$ and $40 \times$ were considered. The generated pair-end read data was aligned to the reference genome with BWA-MEM [23]. We set the repeat number on the root of the generated coalescent trees to 25 to obtain repeat numbers with the size close to the read length. For coalescentSTR and the basic model, read pairs satisfying the following conditions were extracted for obtaining the insert size data for each individual:

- Directions of paired-end reads are concordant.

- The start position of the forward read in each aligned read pair is located before the start position of the STR region.

- The end position of the reverse read in each aligned read pair is located after the end position of the STR region.

Let $n_{i_{1}}$ and $n_{i_{2}}$ be true repeat numbers for the $i$ th individual. We also let $\hat{n}_{i_{1}}$ and $\hat{n}_{i_{2}}$ be estimated repeat numbers for the $i$ th individual. For the evaluation, we considered 
a root mean squared error (RMSE) between true and estimated repeat numbers given by

$\sqrt{\frac{1}{2 I} \sum_{i=1}^{I} \min \left\{\left(n_{i_{1}}-\hat{n}_{i_{1}}\right)^{2}+\left(n_{i_{2}}-\hat{n}_{i_{2}}\right)^{2},\left(n_{i_{1}}-\hat{n}_{i_{2}}\right)^{2}+\left(n_{i_{2}}-\hat{n}_{i_{1}}\right)^{2}\right\}}$.

We evaluated the performance of coalescentSTR, the basic model, lobSTR [8], RepeatSeq [9], and STRViper [10]. In coalescentSTR, an STR mutation rate was selected from rates in $\{0.01,0.1,0.5,0.75,1,1.2,2,5,10,100\}$ multiplied by the true STR mutation rate based on the value given in Eq. (7). $n_{\max }$ and $n_{\min }$ were set to 40 and zero, respectively. For each condition, we prepared ten coalescent trees and generated sequence datasets from them. In order to examine the effect of considering the genealogy, we randomly shuffled haplotypes on phased genotypes and used them for coalescentSTR. Tables 1 and 2 show RMSE values for results from coalescentSTR, coalescentSTR with the shuffled haplotypes (coalescentSTR shuffled), the basic model, lobSTR, RepeatSeq, and STRViper averaged on ten trials for the five types of individual counts, STR mutation rates of $2.73 \times 10^{-4}$ and $1.00 \times 10^{-3}$, and read coverages of $20 \times$ and $40 \times$, respectively.

If no STR variant was detected, the reference repeat number, 17, was assigned as the estimated repeat number. STRViper reports only one repeat number for each individuals although each individual has two repeat numbers. Thus, two repeat numbers in each individual were set to the same value in results of STRViper. CoalescentSTR gives the best result in most of the conditions and coalescentSTR (shuffled) gives the best result in some conditions with sample sizes of 5 and 10. If the sample size considered for estimation is small, the improvement of the performance by considering multiple individuals in coalescentSTR is limited. Thus, coalescentSTR (shuffled) can provide better results than coalescentSTR for some conditions with small sample sizes.
Since some repeat numbers are longer than or equal to the read length, the results from paired-end read distance based methods (coalescentSTR, basic model, and STRViper) are better than those from methods counting repeat numbers in sequences reads (lobSTR and RepeatSeq). The RMSE value is smaller for considering more individuals on coalescentSTR. In addition, the performance of coalescentSTR with the shuffled haplotypes is worse than that of coalescentSTR with correct haplotypes. These observations support the effectiveness of considering the genealogy. The RMSE value for each method on the dataset with read coverage of $40 \times$ is smaller than that on the dataset with read coverage of $20 \times$ in most of the cases.

Figure 2 (a), (b), (c), (d), (e), and (f) show plots for comparing the sum of estimated diploid repeat numbers for one individual and the sum of corresponding true diploid repeat numbers for coalescentSTR, coalescentSTR (shuffled), Basic Model, lobSTR, RepeatSeq, and STRViper, respectively. The simulation datasets with STR mutation rate of $1.00 \times 10^{-3}$ and read coverage of $40 \times$ are used in the plots. In each plot, the $\mathrm{x}$-axis indicates the sum of true diploid repeat numbers for one individual and the $y$-axis indicates the sum of estimated diploid repeat numbers. Ideally, points in plots are located on the diagonal line.

In the plot for coalescentSTR, points are around the diagonal line. Points in the plot for coalescentSTR (shuffled) are also located around the diagonal line, but scattered in larger area than those in the plot for coalescentSTR. In addition, points in the plot for the basic model are scattered in larger area around the diagonal line than those in plots for coalescentSTR and coalescentSTR (shuffled). There exists a horizontal line with the value twice as much as the reference repeat number in plots for lobSTR and RepeatSeq The line is due to points for cases where these methods failed to STR variants and provided the reference repeat number as estimated repeat numbers. For cases with STR variants which can be detected by RepeatSeq, the corresponding points

Table 1 Comparison of estimated repeat numbers in terms of RMSE for simulation datasets with STR mutation rates of $2.73 \times 10^{-4}$ and $1.00 \times 10^{-3}$ and read coverage of $20 \times$

\begin{tabular}{|c|c|c|c|c|c|c|c|c|c|c|}
\hline \multirow{2}{*}{$\begin{array}{l}\text { STR mutation rate } \\
\text { No. of samples }\end{array}$} & \multicolumn{5}{|c|}{$2.73 \times 10^{-4}$} & \multicolumn{5}{|c|}{$1.00 \times 10^{-3}$} \\
\hline & 5 & 10 & 20 & 50 & 100 & 5 & 10 & 20 & 50 & 100 \\
\hline CoalescentSTR & 2.30 & 2.46 & 2.17 & 1.25 & 1.09 & 4.09 & 3.22 & 2.24 & 1.96 & 1.81 \\
\hline CoalescentSTR (shuffled) & 2.37 & 2.42 & 2.30 & 1.38 & 1.96 & 4.02 & 3.52 & 3.05 & 2.73 & 3.33 \\
\hline Basic Model & 4.18 & 5.37 & 5.00 & 5.15 & 4.91 & 5.39 & 5.11 & 5.41 & 4.94 & 5.03 \\
\hline lobSTR & 9.09 & 7.33 & 6.02 & 7.47 & 5.94 & 10.1 & 7.04 & 5.13 & 6.67 & 6.20 \\
\hline RepeatSeq & 9.09 & 7.33 & 6.02 & 7.47 & 5.91 & 10.1 & 7.03 & 5.09 & 6.66 & 6.18 \\
\hline STRViper & 8.38 & 6.87 & 5.57 & 6.91 & 5.60 & 9.37 & 6.59 & 4.90 & 6.23 & 5.75 \\
\hline
\end{tabular}

Repeat numbers were estimated with datasets with 5, 10,20,50, and 100 individuals. The best result in each condition is in bold 
Table 2 Comparison of estimated repeat numbers in terms of RMSE for simulation datasets with STR mutation rates of $2.73 \times 10^{-4}$ and $1.00 \times 10^{-3}$ and read coverage of $40 \times$

\begin{tabular}{|c|c|c|c|c|c|c|c|c|c|c|}
\hline \multirow{2}{*}{$\begin{array}{l}\text { STR mutation rate } \\
\text { No. of samples }\end{array}$} & \multicolumn{5}{|c|}{$2.73 \times 10^{-4}$} & \multicolumn{5}{|c|}{$1.00 \times 10^{-3}$} \\
\hline & 5 & 10 & 20 & 50 & 100 & 5 & 10 & 20 & 50 & 100 \\
\hline CoalescentSTR & 2.47 & 2.20 & 1.86 & 1.11 & 1.00 & 3.28 & 2.75 & 2.18 & 1.74 & 1.61 \\
\hline CoalescentSTR (shuffled) & 2.58 & 2.32 & 2.16 & 1.38 & 1.94 & 3.66 & 3.36 & 2.91 & 2.73 & 3.03 \\
\hline Basic Model & 4.18 & 4.48 & 4.39 & 4.18 & 4.07 & 4.74 & 4.09 & 4.33 & 4.17 & 4.19 \\
\hline lobSTR & 9.09 & 7.32 & 6.02 & 7.47 & 5.94 & 10.10 & 7.05 & 5.12 & 6.67 & 6.20 \\
\hline RepeatSeq & 9.10 & 7.34 & 6.02 & 7.47 & 5.94 & 10.08 & 7.03 & 5.11 & 6.65 & 6.18 \\
\hline STRViper & 7.93 & 6.55 & 5.32 & 6.57 & 5.37 & 8.88 & 6.32 & 4.81 & 5.97 & 5.51 \\
\hline
\end{tabular}

Repeat numbers were estimated with datasets with 5, 10,20,50, and 100 individuals. The best result in each condition is in bold

are located around the diagonal line tightly. On the other hand, points associated with STR variants which can be detected by lobSTR are scattered around the diagonal line. In the plot for STRViper, the sum of estimated diploid repeat numbers is correlated with the sum of true diploid repeat numbers, but differences between estimated repeat numbers and the reference repeat number are underestimated.

\section{Real data analysis}

We evaluated the performance of coalescentSTR, basic model, lobSTR, RepeatSeq, and STRViper using exome (a)

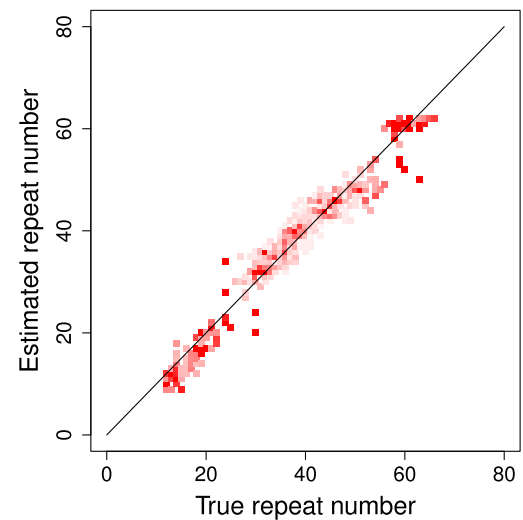

(d)

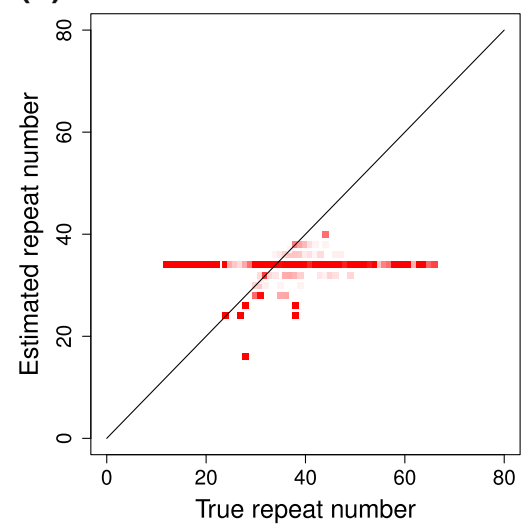

(b)

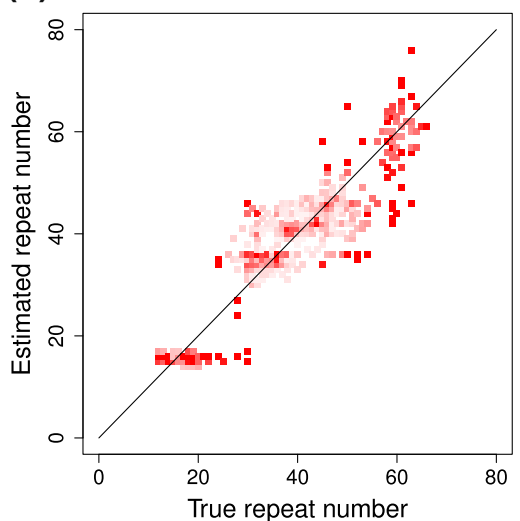

(e)

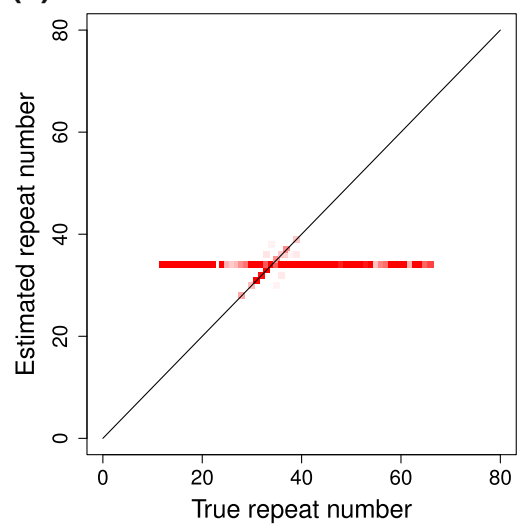

(c)

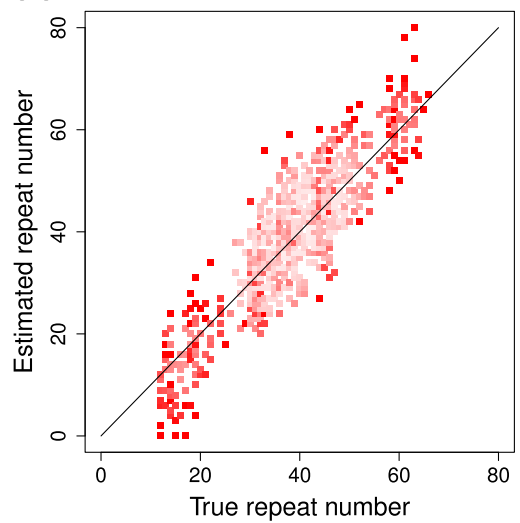

(f)

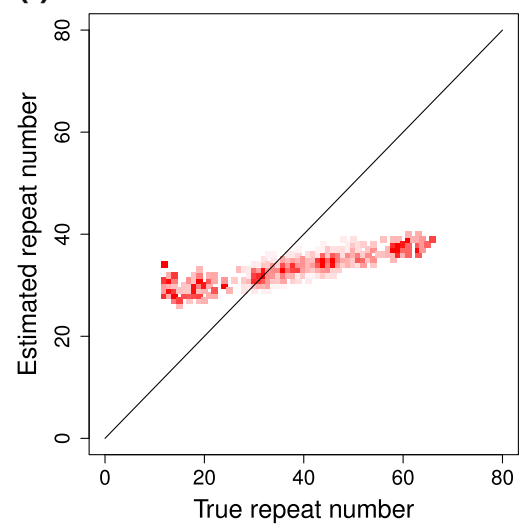

Fig. 2 A plot comparing sums of true repeat numbers and estimated repeat numbers for each individual on simulation data with mutation rate of $1.00 \times 10^{-3}$ and read coverage of $40 \times$. The $x$-axis and $y$-axis indicate the sum of true diploid repeat numbers and the sum of estimated diploid repeat numbers for one individual, respectively. Plots for coalescentSTR, coalescentSTR (shuffled), Basic Model, lobSTR, RepeatSeq, and STRViper are receptively in $(\mathbf{a}),(\mathbf{b}),(\mathbf{c}),(\mathbf{d}),(\mathbf{e})$, and $(\mathbf{f})$ 
sequencing data of JPT individuals and WGS data of CEU and GBR individuals.

\section{Performance evaluation with exome sequencing data}

We evaluated the performance of coalescentSTR, basic model, lobSTR, RepeatSeq, and STRViper on an STR region comprised of TCA repeats located in the exon region of CENPP at chr9:95237025-95237069 by using 1KGP exome datasets for 33 HapMap JPT individuals [5]. The reference repeat number is 14 , and repeat numbers in the region range mainly from 11 to 16 . Read length of datasets for some individuals is $100 \mathrm{bp}$ and that for remaining individuals is $75 \mathrm{bp}$. Since the total length of the STR region is included in the read length, repeat numbers can be inferred directly from the sequence reads spanning the region. In order to evaluate performance on repeats with the size close to the read length, we truncated the tail of each read to obtain pairedend sequence datasets with length of 50 bp in FASTQ format. For the true repeat numbers of the datasets, repeat numbers estimated from the original datasets with lobSTR were used. Sequence reads in the obtained datasets were aligned to the reference genome with BWAMEM. Insert size distribution was obtained from the datasets for each individual. Phased genotypes around the STR region for coalescentSTR were obtained from the 1KGP Phase3 imputation panels released in October, 12, 2014 [5]. Phased genotypes at 4,000 bp upstream and downstream positions of the STR region were used for sampling coalescent trees. An STR mutation rate was selected from $\{0.0001,0.0005,0.001,0.005,0.01\}$ based on the value given by Eq. (7). $n_{\max }$ and $n_{\min }$ were set to 40 and zero, respectively. RMSE values in the results from coalescentSTR, coalescentSTR (shuffled), the basic model, lobSTR, RepeatSeq, and STRViper on the datasets with read length of $50 \mathrm{bp}$ are summarized in Table 3. Since read length of $50 \mathrm{bp}$ is not sufficient for the detecting the repeat patterns directly from sequence reads including the STR region, no STR variant was detected in lobSTR and RepeatSeq for all the individuals. Although STRViper assumes the normality on insert size distribution, the actual insert size in the datasets is not normally distributed as shown in Fig. 3, and hence STRViper may fail to detect the STR variants. For cases of detecting no STR variant, the reference repeat number was assigned for the estimated repeat numbers. CoalescentSTR gives the best RMSE, and the basic model gives the worst RMSE.
Since the quality of the data is different between datasets, the basic model failed to estimate repeat numbers for low quality datasets. In addition, since the true repeat numbers do not vary a lot, the results with the reference repeat number for all the individuals give not so bad RMSE, and hence the result of the basic model is the worst among the methods.

Figure 4 (a), (b), (c), (d), (e), and (f) show plots for comparing the sum of estimated diploid repeat numbers for one individual and the sum of corresponding true diploid repeat numbers for coalescentSTR, coalescentSTR (shuffled), the basic model, lobSTR, RepeatSeq, and STRViper for real data set, respectively.

In the plot for coalescentSTR, points are located around the diagonal line. Points in the plot for coalescentSTR (shuffled) are also located around the diagonal line, but scattered in larger area than those in the plot for coalescentSTR. In the plot for the basic model, the sum of estimated repeat numbers is not correlated with the sum of true repeat numbers because the amount data for each individual in this experiment is not sufficient for estimating repeat numbers correctly. Since lobSTR, RepeatSeq, and STRViper could not detect STR variants for any sample, only a horizontal line with the value twice as much as the reference repeat number is observed in the plots for lobSTR, RepeatSeq, and STRViper.

\section{Performance evaluation with WGS data}

We applied coalescentSTR and other existing methods to WGS data of a HapMap CEU individual, NA12878 from HiSeq 2000 to estimate numbers of CAC repeats at chr1:20200573-20200666 in GRCh37 for NA12878. Read length and average insert size of the WGS data are respectively $101 \mathrm{bp}$ and $300 \mathrm{bp}$, and its read coverage is $50 \times$. The data was provided by the Illumina Platinum Genomes Project through the European Nucleotide Archive under the study accession PRJEB3381 (http://www.ebi.ac.uk/ ena/data/view/ERP001960). In addition to the WGS data of NA12878, we used WGS data for 35 HapMap CEU and 35 HapMap GBR individuals released in May, 22, 2012 by 1 KGP [5]. Read length and read coverage of the WGS data of these 70 individual is $100 \mathrm{bp}$ and $5 \times$, respectively. Sequence reads in the WGS data of NA12878 were aligned with BWA-MEM while those in the WGS of others were aligned with BWA [24]. Phased genotypes around the STR region for coalescentSTR were obtained from the

Table 3 Comparison of estimated repeat numbers in terms of RMSE for real exome data for JPT individuals in 1KGP

\begin{tabular}{lllllll}
\hline Method & CoalescentSTR & $\begin{array}{l}\text { CoalescentSTR } \\
\text { (shuffled) }\end{array}$ & Basic model & lobSTR & RepeatSeq & STRViper \\
\hline RMSE & $\mathbf{1 . 3 3}$ & 2.38 & 9.44 & 1.63 & 1.63 & 1.63 \\
\hline
\end{tabular}

The best result is in bold 


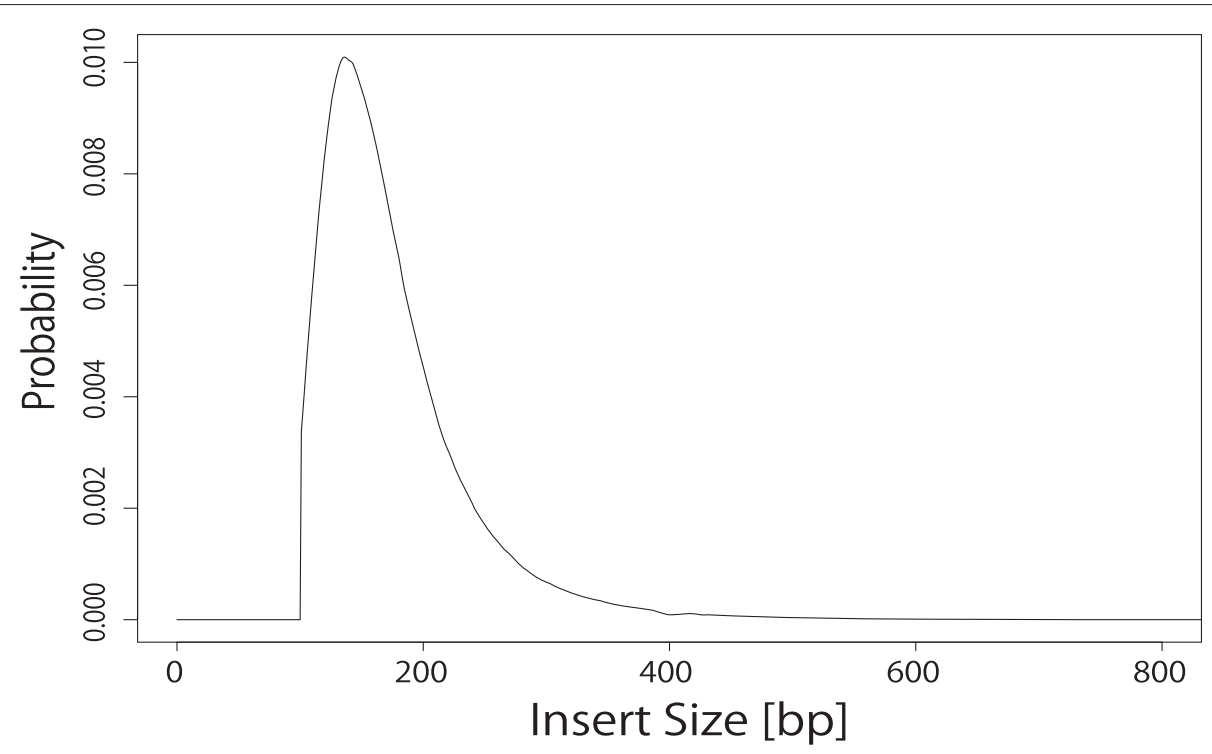

Fig. 3 An empirically obtained insert size distribution. This empirical distribution was generated from aligned read pairs in exome data of a JPT individual in 1 KGP sequenced with Illumina HiSeq

(a)

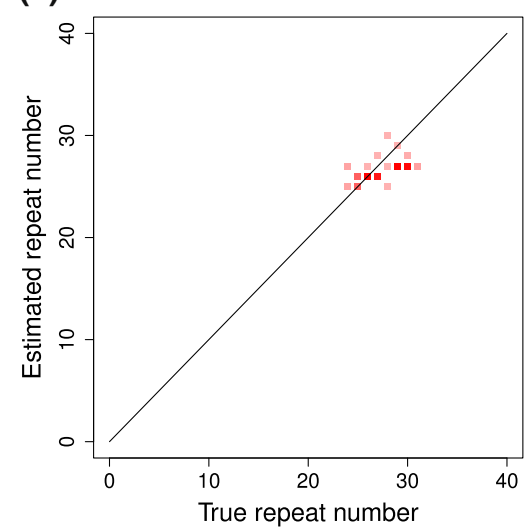

(d)

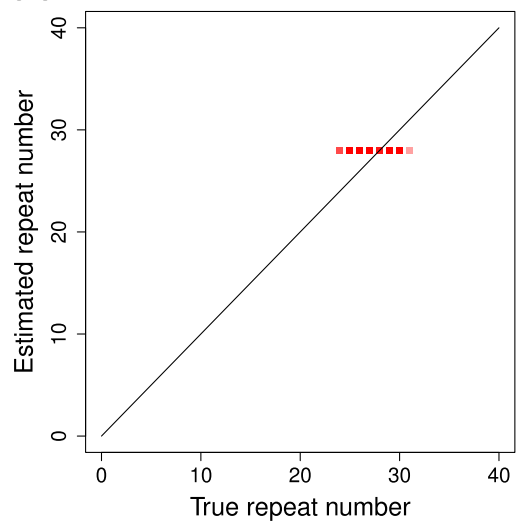

(b)

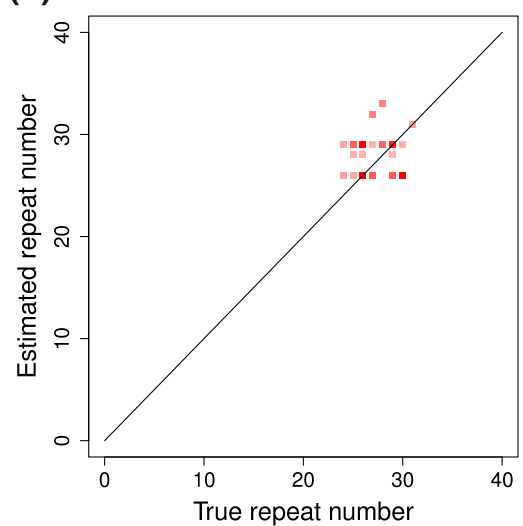

(e)

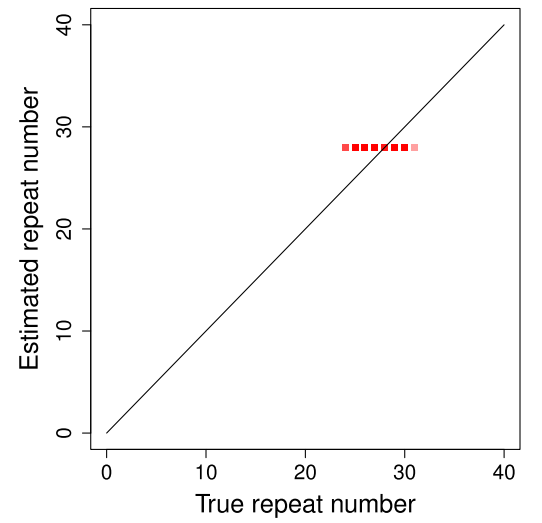

(c)

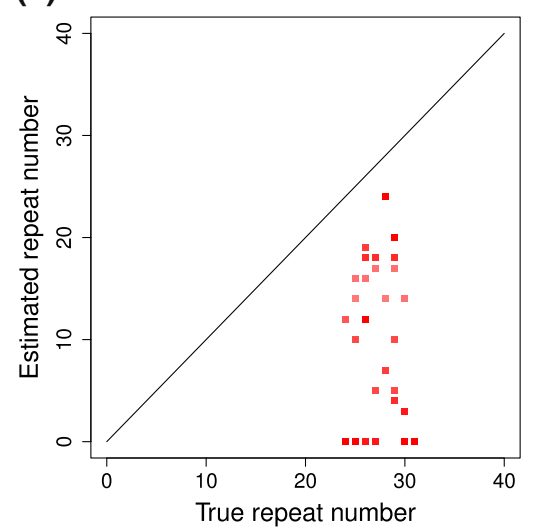

(f)

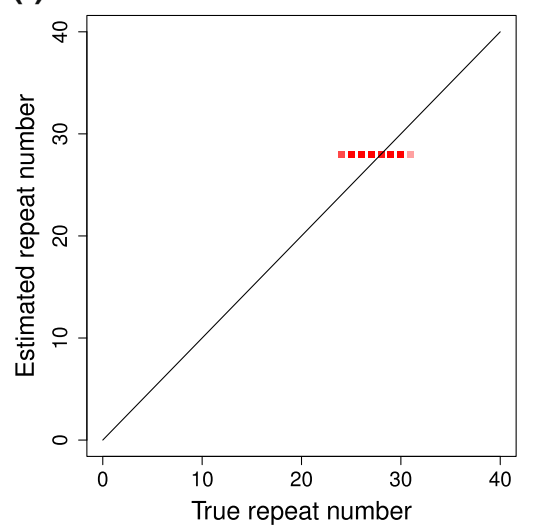

Fig. 4 A plot comparing sums of true repeat numbers and estimated repeat numbers for each individual on real data. The $x$-axis and $y$-axis indicate the sum of true diploid repeat numbers and the sum of estimated diploid repeat numbers for one individual, respectively. Plots for coalescentSTR, coalescentSTR (shuffled), Basic Model, lobSTR, RepeatSeq, and STRViper are receptively in $(\mathbf{a}),(\mathbf{b}),(\mathbf{c}),(\mathbf{d}),(\mathbf{e})$, and (f) 
1KGP Phase3 imputation panels released in October, 12, 2014 [5]. For sampling coalescent trees, phased genotypes at 3,000 bp upstream and downstream positions of the STR region were used. An STR mutation rate was selected from $\{0.0001,0.0005,0.001,0.005,0.01\}$ based on the value given by Eq. (7). $n_{\max }$ and $n_{\min }$ were set to 40 and zero, respectively.

The size of the STR region is $93 \mathrm{bp}$ in the reference genome, and it is difficult to estimate repeat numbers in the region by directly counting repeat numbers in the aligned reads for the data of read length of 100 bp due to the lack of aligned bases to the flanking regions in spanning reads. Thus, we estimated repeat numbers estimated from high coverage sequencing data with long reads, and used the estimated repeat numbers as true repeat numbers for the evaluation. For sequencing data with long reads, PacBio sequencing data for NA12878 provided from GIAB Reference Materials and Data (ftp://ftp-trace.ncbi.nlm.nih.gov/giab/ftp/data/ NA12878/NA12878_PacBio_MtSinai) [25] was used, and repeat numbers were estimated by the following procedures:

- Error-corrected reads with Falcon (https://github. com/PacificBiosciences/FALCON) in FASTA format were aligned to GRCh37 with BWA-MEM.

- The number of bases aligned in the STR region was counted for each read spanning the region.

- Two-component Gaussian mixture model was applied to the set of numbers of bases obtained the above, and estimated means for two components divided by the size of the repeat pattern were adopted as estimated repeat numbers.

The estimated repeat numbers from the above procedures were 28.02 and 25.38 and used for the evaluation by calculating RMSE given as follows:

$\sqrt{\frac{1}{2} \min \left\{\left(28.02-\hat{n}_{1}\right)^{2}+\left(25.38-\hat{n}_{2}\right)^{2},\left(28.02-\hat{n}_{2}\right)^{2}+\left(25.38-\hat{n}_{1}\right)^{2}\right\}}$,

where $\hat{n}_{1}$ and $\hat{n}_{2}$ are estimated repeat numbers for NA12878. The estimated repeat numbers from coalescentSTR, coalescentSTR (shuffled), the basic model, lobSTR, RepeatSeq, and STRViper and their corresponding RMSE values with the repeat numbers estimated from the PacBio data are summarized in Table 4. Since no variant was detected by lobSTR and RepeatSeq, the reference repeat number, 31, was assigned to their estimated results. Similarly to the results in the former real data experiment, coalescentSTR gives the best RMSE and the basic model gives the worst RMSE.

\section{Comparison of computational time}

Table 5 shows the computational time of coalescentSTR, the basic model, lobSTR, RepeatSeq, and STRViper for
Table 4 Estimated repeat numbers from WGS data from HiSeq 2000 for NA12878 and their corresponding RMSE values with the repeat numbers estimated from PacBio sequencing data for NA12878

\begin{tabular}{lll}
\hline Method & Estimated repeat numbers & RMSE \\
\hline CoalescentSTR & $28 / 26$ & $\mathbf{0 . 4 4}$ \\
CoalescentSTR (shuffled) & $25 / 25$ & 2.15 \\
Basic Model & $33 / 32$ & 5.46 \\
lobSTR & $31 / 31$ & 4.50 \\
RepeatSeq & $31 / 31$ & 4.50 \\
STRViper & $31.12 / 31.12$ & 4.61 \\
\hline
\end{tabular}

The best result is in bold

the simulation data in Section Simulation analysis and the real data for HapMap JPT individuals in Section Performance evaluation with exome sequencing data. For simulation data, the dataset with 100 individuals and STR mutation rate of $2.73 \times 10^{-4}$ is considered. Note that read alignment time with lobSTR is not included for the computational time of lobSTR because read alignment time with BWA-MEM is not included in those of other algorithms. All the computation was performed on Intel Xeon CPU E5-2670 processors with single thread. CoalescentSTR and the basic model are implemented in Java. For coalescentSTR, computation time for sampling coalescent trees and estimation using sampled trees are separated in Table 5 as coalescentSTR (sampling) and coalescentSTR (estimation). In both simulation and real data, coalescentSTR requires the most computational time, especially in sampling coalescent trees. Computational time required for estimation by coalescentSTR is slightly more than that of STRViper. For memory consumption, coalescentSTR requires less than 4GB in both sampling and estimation.

Table 5 Comparison of computational time on the simulation dataset with 100 individuals, STR mutation rate of $2.73 \times 10^{4}$, and read coverage of $40 \times$ and the real dataset for HapMap JPT individuals

\begin{tabular}{lrr}
\hline Method & $\begin{array}{c}\text { Computational time } \\
\text { (simulation data) }\end{array}$ & $\begin{array}{c}\text { Computational time } \\
\text { (real data) }\end{array}$ \\
\hline CoalescentSTR (sampling) & $13372.96[\mathrm{~s}]$ & $846.60[\mathrm{~s}]$ \\
CoalescentSTR (estimation) & $452.59[\mathrm{~s}]$ & $66.86[\mathrm{~s}]$ \\
Basic Model & $49.58[\mathrm{~s}]$ & $16.18[\mathrm{~s}]$ \\
lobSTR & $2.72[\mathrm{~s}]$ & $8.17[\mathrm{~s}]$ \\
RepeatSeq & $10.24[\mathrm{~s}]$ & $14.78[\mathrm{~s}]$ \\
STRViper & $407.03[\mathrm{~s}]$ & $83.45[\mathrm{~s}]$ \\
\hline
\end{tabular}




\section{Conclusions}

We proposed a statistical approach named coalescentSTR to estimate repeat numbers in an STR region for multiple individuals from insert size data obtained by pairedend reads in HTS data. We considered the genealogy of the multiple individuals and used the genealogy for propagating repeat number information from insert size among individuals to achieve more accurate estimation of repeat numbers. We evaluated the performance of coalescentSTR, the basic model, lobSTR, RepeatSeq, and STRViper from simulation data and real data from 1KGP and verified the effectiveness of coalescentSTR for STR regions longer than or equal to the read length.

For computational time, coalescentSTR requires the most computational time from the comparison with other existing methods, and its computational time is mainly taken by sampling coalescent trees with MCMC. The use of MCMC with approximate Bayesian computation $(A B C)[26]$ is a solution addressing this issue because the calculation of likelihood for each sampled tree is avoided with $\mathrm{ABC}$ and the calculation mainly requires the computational time for sampling. For larger size of genome structural variations such as large size copy number variations, the recombination of genomes needs to be considered although the recombination is basically not considered in coalescent theory. We are considering to extend the proposed model in future work in order to use ancestral recombination graph, which can handle the recombination in the genealogy of multiple individuals unlike coalescent tree.

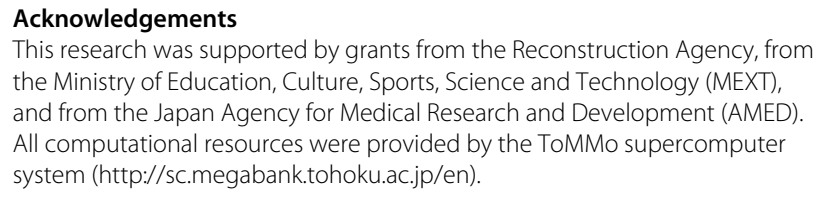
the Ministry of Education, Culture, Sports, Science and Technology (MEXT), and from the Japan Agency for Medical Research and Development (AMED). All computational resources were provided by the ToMMo supercomputer system (http://sc.megabank.tohoku.ac.jp/en).

\section{Declarations}

This article has been published as part of BMC Genomics Volume 17 Supplement 5, 2016. Selected articles from the 11th International Symposium on Bioinformatics Research and Applications (ISBRA '15): genomics. The full contents of the supplement are available online https://bmcgenomics. biomedcentral.com/articles/supplements/volume-17-supplement-5.

\section{Funding}

Publication costs for this work were funded by the Tohoku Medical Megabank Project (Special Account for reconstruction from the Great East Japan Earthquake).

\section{Availability of data and materials}

Sequence reads from HiSeq 2000 for NA12878 are available at the European Nucleotide Archive under the study accession number PRJEB3381 (http:// www.ebi.ac.uk/ena/data/view/ERP001960). Sequence reads from PacBio for NA12878 are available in GIAB Reference Materials and Data through NCBI FTP site (ftp://ftp-trace.ncbi.nlm.nih.gov/giab/ftp/data/NA12878/NA12878_ PacBio_MtSinai). Sequence reads for 33 HapMap JPT individuals, 35 CEU individuals, and 35 GBR individuals are available from the 1000 Genomes Project through EBI FTP site (ftp://ftp.1000genomes.ebi.ac.uk/vol1/ftp/).

\section{Authors' contributions}

KK proposed the statistical model and implemented the program for evaluation. YK provided the knowledge and advice about coalescent theory and computational techniques on the field. KK, NN, TM, and MN developed fundamental environments for the evaluation using simulation and real data studies. KK, YK, NN, TM, TH, MN carefully checked equations and other contents in this manuscript. All authors read and approved the final manuscript.

\section{Competing interests}

The authors declare that they have no competing interests.

\section{Consent for publication}

Not applicable.

\section{Ethics approval and consent to participate}

Not applicable.

\section{Author details}

${ }^{1}$ Tohoku Medical Megabank Organization, Tohoku University, 2-1, Seiryo-machi, Aoba-ku, 980-8573, Sendai, Japan. ${ }^{2}$ Institute for Genomic Medicine, University of California, San Diego, 9500 Gilman Drive \#0761, 92093-0761, San Diego, USA.

Published: 31 August 2016

\section{References}

1. DePristo MA, Banks E, Poplin R., Garimella KV, Maguire JR, Hartl C, Philippakis AA, del Angel G, Rivas MA, Hanna M, McKenna A, Fennell TJ, Kernytsky AM, Sivachenko AY, Cibulskis K, Gabriel SB, Altshuler D, Daly MJ. A framework for variation discovery and genotyping using next-generation dna sequencing data. Nat Genet. 2011;43:491-8.

2. Kojima K, Nariai N, Mimori T, Takahashi M, Yamaguchi-Kabata Y, Sato Y, Nagasaki M. A statistical variant calling approach from pedigree information and local haplotyping with phase informative reads. Bioinformatics. 2013;29(22):2835-43.

3. Kojima K, Nariai N, Mimori T, Yamaguchi-Kabata Y, Sato Y, Kawai Y, Nagasaki M. Hapmonster: a statistically unified approach for variant calling and haplotyping based on phase-informative reads. Lect Nodes Comput Sci. 2014;8542:107-18.

4. Li H, Ruan J, Durbin R. Mapping short dna sequencing reads and calling variants using mapping quality scores. Genome Res. 2008;18(11):1851-8.

5. 1000 Genomes Project Consortium. An integrated map of genetic variation from 1,092 human genomes. Nature. 2012;491(7422):56-65.

6. Mimori T, Nariai N, Kojima K, Takahashi M, Ono A, Sato Y, Yamaguchi-Kabata Y, Kawai Y, Nagasaki M. iSVP: an integrated structural variant calling pipeline from high-throughput sequencing data. BMC Syst. Biol. 2013;7(Suppl 6):S8.

7. Walker FO. Huntington's disease. Lancet. 2007;369(9557):2185-228.

8. Gymrek M, Golan D, Rosset S, Erlich Y. lobstr: A short tandem repeat profiler for personal genomes. Genome Res. 2012;6:1154-62.

9. Highnam G, Franck C, Martin A, Stephens C, Puthige A, Mittelman D. Accurate human microsatellite genotypes from high-throughput resequencing data using informed error profiles. Nucleic Acids Res. 2013;41(1).

10. Cao MD, Tasker E, Willadsen K, Imelfort M, Vishwanathan S, Sureshkumar S, Balasubramanian S, Boden M. Inferring short tandem repeat variation from paired-end short reads. Nucleic Acids Res. 2014;42(3)

11. Kingman JFC. On the genealogy of large populations. J Appl Probab. 1982;19(A):27-43.

12. Tajima F. Evolutionary relationship of dna sequences in finite populations. Genetics. 1983;105:437-60.

13. Wakeley J. Coalescent Theory: An Introduction. Greenwood Village: Roberts and Company Publishers; 2008

14. Yedidia JS, Freeman WT, Weiss Y. Constructing free-energy approximations and generalized belief propagation algorithms. IEEE Trans Inf. 2005;51(7):2282-312.

15. Liu Q, Ihler A. Variational algorithms for marginal map. J Mach Learn Res. 2013;14:3165-200.

16. Nachman MW, Crowell SL. Estimate of the mutation rate per nucleotide in humans. Genetics. 2000;156(1):297-304. 
17. Sun JX, Helgason A, Masson G, Ebenesersdóttir SS, Li H, Mallick S, Gnerre S, Patterson N, Kong A, Reich D, Stefansson K. A direct characterization of human mutation based on microsatellites. Nat Genet. 2012;44:1161-5.

18. Hudson RR. Gene genealogies and the coalscent process In: Harris TJR, editor. Oxford Surveys in Evolutionary Biology. Stoner G(Series Editor): Methods and Perspectives in Cell Biology, vol 1. New York: Academic Press; 1990. p. 1-44.

19. Zhivotovsky LA, Feldman MW. Microsatellite variability and genetic distances. Proc Natl Acad Sci. 1995;92(6):11549-52.

20. Tavaré S. Part I: Ancestral inference in population genetics. Lect Nodes Math. 2004;1837:1-188.

21. Yu N, Chen FC, Ota S, Jorde LB, Pamilo P, Patthy L, Ramsay M, Jenkins T, Shyue SK, Li WH. Larger genetic differences within africans than between africans and eurasians. Genetics. 2002;161:269-74.

22. Benson G. Tandem repeats finder: a program to analyze dna sequences. Nucleic Acids Res. 1999;27(2):573-80.

23. Li H. Aligning sequence reads, clone sequences and assembly contigs with bwa-mem. 2013. https://arxiv.org/abs/1303.3997.

24. Li H, Durbin R. Fast and accurate short read alignment with burrows-wheeler transform. Bioinformatics. 2009;25(14):1754-60.

25. Zook JM, Chapman B, Wang J, Mittelman D, Hofmann O, Hide W, Salit $M$. Integrating human sequence data sets provides a resource of benchmark snp and indel genotype calls. Nat Biotechnol. 2014;32:246-51.

26. Marjoram P, Molitor J, Plagnol V, Tavaré S. Markov chain monte carlo without likelihoods. Proc Natl Acad Sci. 2003;100(26):15324-8.

\section{Submit your next manuscript to BioMed Central and we will help you at every step:}

- We accept pre-submission inquiries

- Our selector tool helps you to find the most relevant journal

- We provide round the clock customer support

- Convenient online submission

- Thorough peer review

- Inclusion in PubMed and all major indexing services

- Maximum visibility for your research

Submit your manuscript at www.biomedcentral.com/submit 